\title{
Transverse centroid oscillations in solenoidially focused beam transport lattices
}

\author{
Steven M. Lund \\ Lawrence Livermore National Laboratory, Livermore, CA 94550, USA \\ Christopher J. Wootton and Edward P. Lee \\ Lawrence Berkeley National Laboratory, Berkeley, CA 94720, USA
}

\begin{abstract}
Linear equations of motion are derived that describe small-amplitude centroid oscillations induced by displacement and rotational misalignments of the focusing solenoids in the transport lattice, dipole steering elements, and initial centroid offset errors. These equations are analyzed in a local rotating Larmor frame to derive complex-variable "alignment functions" and "bending functions" that efficiently describe the characteristics of the centroid oscillations induced by mechanical misalignments of the solenoids and dipole steering elements. The alignment and bending functions depend only on properties of the ideal lattice in the absence of errors and steering and have associated expansion amplitudes set by the misalignments and steering fields. Applications of this formulation are presented for statistical analysis of centroid deviations, calculation of actual lattice misalignments from centroid measurements, and optimal beam steering.
\end{abstract}

Key words: solenoid, transport, centroid, steering, alignment

PACS: 29.27.Bd, 41.75.-i, 41.85.-p, 52.59.Sa

\section{Introduction}

Solenoidal focusing is employed in a variety of ion and electron beam transport applications such as ion beam driven experiments in warm dense matter[1], intense electron beams for driving flash x-ray radiography[2], and electron cooling for hadron beams $[3,4]$. Such applications typically require precise control of the beam centroid to: control the placement of the beam spot on target, maintain alignment of the beam with the field in cooling applications, and control a variety of deleterious processes that are enhanced with increasing amplitude of centroid oscillations in the machine. Effects enhanced include nonlinear image charges and currents, applied field nonlinearities, and corkscrew effects $[5,6]$.

Email addresses: SMLund@llnl.gov (Steven M. Lund) 
When image and nonlinear effects are negligible, the transverse centroid orbit of a solenoidally focused beam can be decomposed into two parts: an overall angular "Larmor" rotation about the solenoidal field with a rate of angular phase accumulation proportional to the axial field[7, 8, 9], and superimposed, $x-y$ plane-decoupled betatron oscillations $[10,7,11]$ within the local rotating frame. These combined effects can result in laboratory-frame centroid orbit oscillations with intricate structure.

The centroid oscillations are driven by mechanical alignment errors of the solenoids in the focusing lattice. To leading order, displacement and rotational misalignments of the solenoids generate driving dipole terms that bend an aligned centroid orbit away from the design axis of the machine[12]. The direction that the bending dipole fields act depend on the (typically random) orientation of the misalignments encountered. In the absence of corrections, this tends to increase the centroid oscillation amplitude in a random walk sense proportional to the square root of the number of misaligned solenoids traversed.

Transverse steering corrections of the beam centroid in solenoidal transport systems can be challenging due to the $x-y$ plane coupling of the centroid orbit and typically limited provision for steering and diagnostics in the lattice. Steering dipoles inserted in the lattice typically act with fixed orientation in the laboratory frame and misalignment driven centroid oscillations (if not physically removed) will further accumulate in any solenoids placed between steering dipole pairs necessary to synthesize a correction.

Using an analogy to the "dispersion" function employed to characterize single-particle orbit changes in a lattice in response to momentum spread[10, 9], complex-variable "alignment functions" and "bending functions" are derived that characterize the small-amplitude evolution of the centroid orbit in response to alignment errors and applied dipole steering fields. The centroid orbit is expanded in terms of the alignment and bending functions superimposed on the ideal centroid orbit in the absence of errors or steering. The alignment and bending functions are defined in a manner where they can be calculated based only on properties of the ideal, perfectly aligned lattice and the expansion amplitudes relate to the misalignments and steering fields. This formulation gives insight on the structure of the centroid orbit and is applied to develop procedures to steer the centroid and to calculate solenoid misalignment parameters using measured centroid data and thereby enable optimal centroid correction procedures.

The organization of this paper is the following. Fields of a misaligned solenoid and a dipole steering element of a transport lattice are first characterized in Sec. 2. These field descriptions are employed in Sec. 3 to derive linear equations of motion describing the beam centroid. The centroid orbit is systematically expanded in terms of alignment and bending functions which describe the centroid response to system misalignments and dipole steering fields. Applications of this centroid expansion are presented in Sec. 4 to develop procedures to steer the beam centroid and to calculate actual lattice misalignment parameters. Finally, concluding discussions in Sec. 5 outline limitations of the methodology and readily achievable generalizations. 


\section{Solenoid and dipole field models}

We consider the magnetic field $\mathbf{B}(\mathbf{x})$ of an individual solenoid magnet used for beam focusing. The solenoid is oriented along the longitudinal $z$-axis with $z=0$ corresponding to the axial mid-plane of the magnet and is assumed invariant under azimuthal rotations about the $z$-axis. Consistent with the static, vacuum Maxwell equations, the magnetic field can be expanded in terms of the on-axis axial field of the solenoid $B_{z 0}(z) \equiv B_{z}\left(\mathbf{x}_{\perp}=0, z\right)$ and the transverse coordinate $\mathbf{x}_{\perp}=x \hat{\mathbf{x}}+y \hat{\mathbf{y}}$ as $[11,12]$

$$
\begin{aligned}
& \mathbf{B}_{\perp}(\mathbf{x})=\frac{1}{2} \sum_{\nu=1}^{\infty} \frac{(-1)^{\nu}}{\nu !(\nu-1) !} \frac{\partial^{2 \nu-1} B_{z 0}(z)}{\partial z^{2 \nu-1}}\left(\frac{\left|\mathbf{x}_{\perp}\right|}{2}\right)^{2 \nu-2} \mathbf{x}_{\perp} \\
& B_{z}(\mathbf{x})=B_{z 0}(z)+\sum_{\nu=1}^{\infty} \frac{(-1)^{\nu}}{(\nu !)^{2}} \frac{\partial^{2 \nu} B_{z 0}(z)}{\partial z^{2 \nu}}\left(\frac{\left|\mathbf{x}_{\perp}\right|}{2}\right)^{2 \nu} .
\end{aligned}
$$

For small transverse excursions in $\mathbf{x}_{\perp}$, only leading order terms in the expansion (1) are retained giving

$$
\begin{aligned}
\mathbf{B}_{\perp} & =-\frac{1}{2} \frac{\partial B_{z 0}(z)}{\partial z} \mathbf{x}_{\perp}, \\
B_{z} & =B_{z 0}(z) .
\end{aligned}
$$

Note that the field specified by $B_{z 0}(z)$ in the truncated expansion (2) is divergence free, satisfying $\nabla \cdot \mathbf{B}=0$.

The function $B_{z 0}(z)$ is set by the magnet design and the corresponding field description in Eq. (2) is ideal in the sense that it does not take into account effects such as leads and construction errors that can break azimuthal symmetry. In a realistic beam transport system, the magnet will be placed in a lattice with finite displacement and rotational mechanical alignment errors. We choose to first characterize magnet displacements and then rotations within the displaced coordinate system. For present purposes, the natural coordinate system of the ideal magnet is denoted with primes. The ideal field of the solenoid is denoted as $\mathbf{B}^{\prime}\left(\mathbf{x}^{\prime}\right)$, which is specified by the function $B_{z 0}^{\prime}\left(z^{\prime}\right)$ via Eq. (2) with all coordinates and fields primed.

First, the geometric center of the magnet is translationally displaced by $\boldsymbol{\Delta}=\Delta_{x} \hat{\mathbf{x}}+\Delta_{y} \hat{\mathbf{y}}+\Delta_{z} \hat{\mathbf{z}}$ relative to the laboratory frame, giving

$$
\mathrm{x}^{\prime}=\mathrm{x}-\boldsymbol{\Delta},
$$

and

$$
\mathbf{B}(\mathbf{x})=\mathbf{B}^{\prime}(\mathbf{x}-\boldsymbol{\Delta}) .
$$

This geometry is sketched in Fig. 1(a). 

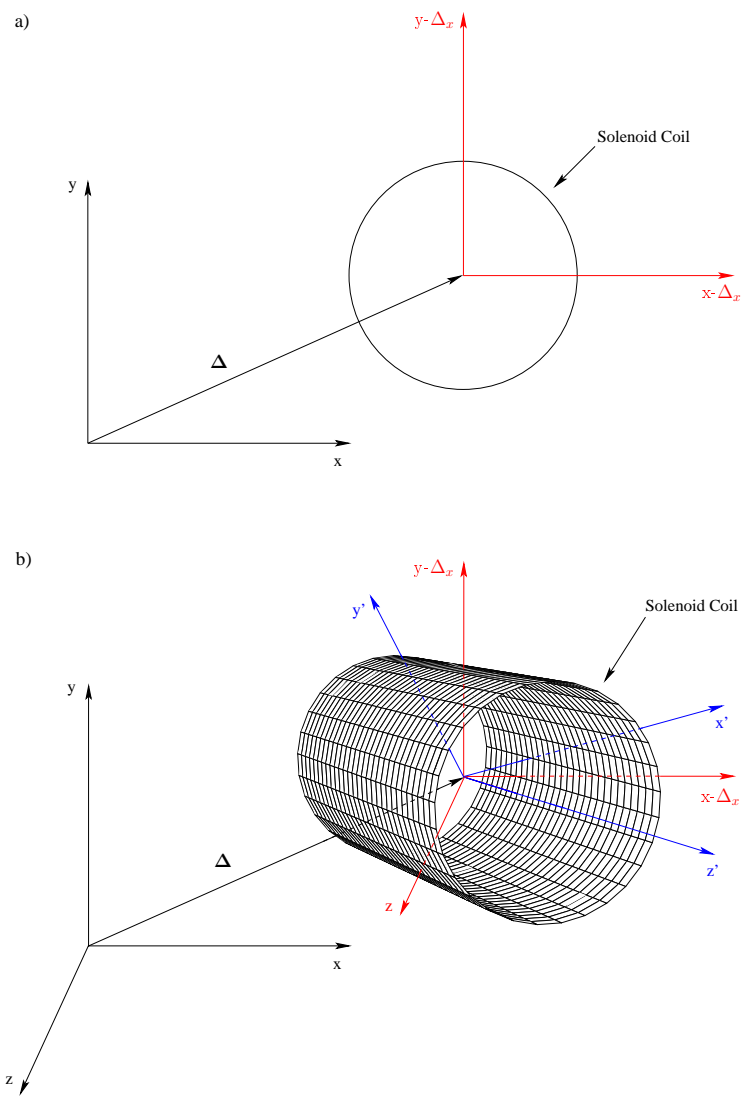

Figure 1: A misaligned solenoid is described by a two-step transformation of an ideal solenoid. First, translation of center by $\boldsymbol{\Delta}$ (a), and then a rotation (b) of the axis of symmetry in the translated system. For clarity, a pure transverse translation $\left(\boldsymbol{\Delta}=\Delta_{x} \hat{\mathbf{x}}+\Delta_{y} \hat{\mathbf{y}}\right)$ is illustrated.

Next, rotational misalignments of the solenoid are accounted for by a second active transformation step where a rotation matrix is applied to the displaced coordinate system as illustrated in Fig. 1(b). Before the rotation, unit vectors in the displaced coordinate system defined by Eq. (3) are parallel to the laboratory frame system (i.e., $\hat{\mathbf{x}}^{\prime}=\hat{\mathbf{x}}$, etc.). Employing spherical coordinates, the solenoid axis of symmetry $\hat{\mathbf{z}}^{\prime}$ is actively rotated through a polar angle $\phi$ and an azimuthal angle $\theta$. Consistently, $\hat{\mathbf{z}}^{\prime}$ is projected into the laboratory frame as

$$
\hat{\mathbf{z}}^{\prime}=\sin \phi \cos \theta \hat{\mathbf{x}}+\sin \phi \sin \theta \hat{\mathbf{y}}+\cos \phi \hat{\mathbf{z}} .
$$

The rotation that defines the orientation of the ideal solenoid axis, $\hat{\mathbf{z}}^{\prime}$, can be thought of as a right-handed rotation through the angle $\phi$ about an axis specified by the unit vector

$$
\hat{\mathbf{s}}=-\sin \theta \hat{\mathbf{x}}+\cos \theta \hat{\mathbf{y}}
$$

All the vectors $\mathbf{v}$ in the translated system can be transformed to the rotated (primed) system of the ideal solenoid by by applying the Rodrigues' Rotation 
Formula

$$
\mathbf{v} \rightarrow \mathbf{v}^{\prime}=\cos \phi \mathbf{v}+(\mathbf{v} \cdot \hat{\mathbf{s}})(1-\cos \phi) \hat{\mathbf{s}}-\sin \phi(\mathbf{v} \times \hat{\mathbf{s}}) .
$$

Using this formula, the unit vectors $\hat{\mathbf{x}}^{\prime}$ and $\hat{\mathbf{y}}^{\prime}$ are projected into the laboratory frame as

$$
\begin{aligned}
& \hat{\mathbf{x}}^{\prime}=\left(\cos \phi \cos ^{2} \theta+\sin ^{2} \theta\right) \hat{\mathbf{x}}+(\cos \phi-1) \sin \theta \cos \theta \hat{\mathbf{y}}-\sin \phi \cos \theta \hat{\mathbf{z}} . \\
& \hat{\mathbf{y}}^{\prime}=(\cos \phi-1) \sin \theta \cos \theta \hat{\mathbf{x}}+\left(\cos \phi \sin ^{2} \theta+\cos ^{2} \theta\right) \hat{\mathbf{y}}-\sin \phi \sin \theta \hat{\mathbf{z}} .
\end{aligned}
$$

Applying Eqs. (3), (5), and (8), the transform relating coordinates (x) of the laboratory frame and the translated and rotated system of the ideal magnet $\left(\mathbf{x}^{\prime}\right)$ can be expressed in matrix form as

$$
\mathbf{x}^{\prime}=\mathbf{R} \cdot(\mathbf{x}-\boldsymbol{\Delta}),
$$

where $\mathbf{R}$ is the orthogonal rotation matrix

$$
\mathbf{R} \equiv\left[\begin{array}{lll}
\cos \phi \cos ^{2} \theta+\sin ^{2} \theta & (\cos \phi-1) \sin \theta \cos \theta & -\sin \phi \cos \theta \\
(\cos \phi-1) \sin \theta \cos \theta & \cos \phi \sin ^{2} \theta+\cos ^{2} \theta & -\sin \phi \sin \theta \\
\sin \phi \cos \theta & \sin \phi \sin \theta & \cos \phi
\end{array}\right] .
$$

Consistent with the azimuthal symmetry of the ideal solenoid in the primed system, note that the rotation matrix $\mathbf{R}$ does not address azimuthal rotations of the solenoid about $\mathbf{z}^{\prime}$. The magnetic field $\mathbf{B}^{\prime}$ of the ideal solenoid can be consistently expressed in the laboratory frame as,

$$
\mathbf{B}^{\prime}\left(\mathbf{x}^{\prime}\right)=\mathbf{R} \cdot \mathbf{B}(\mathbf{x}),
$$

or equivalently as

$$
\mathbf{B}(\mathbf{x})=\mathbf{R}^{T} \cdot \mathbf{B}^{\prime}\left(\mathbf{x}^{\prime}\right) .
$$

Here we employed $\mathbf{R}^{-1}=\mathbf{R}^{T}$ for the orthogonal matrix $\mathbf{R}$, where $\mathbf{R}^{-1}$ and $\mathbf{R}^{T}$ denote the inverse and transpose matrices of $\mathbf{R}$.

For small angular misalignments of the solenoid, $|\phi| \ll 1$, and $\sin \phi$ and $\cos \phi$ can be expanded to leading order as $\cos \phi \simeq 1$ and $\sin \phi \simeq \phi$. Denoting transverse rotation parameters

$$
\begin{aligned}
& \Theta_{x} \equiv \phi \cos \theta, \\
& \Theta_{y} \equiv \phi \sin \theta,
\end{aligned}
$$

the rotation matrix $\mathbf{R}$ in Eq. (10) is given approximately by

$$
\mathbf{R} \simeq\left[\begin{array}{lll}
1 & 0 & -\Theta_{x} \\
0 & 1 & -\Theta_{y} \\
\Theta_{x} & \Theta_{y} & 1
\end{array}\right]
$$

This approximation of $\mathbf{R}$ results in a loss of exact orthogonality of the matrix, which will effectively cause small violations of $\nabla \cdot \mathbf{B}=0$ in the field model. The angle parameters $\Theta_{x}$ and $\Theta_{y}$ can be geometrically interpreted as small 
angle rotations in the $\left(y-\Delta_{y}\right)-\left(z-\Delta_{z}\right)$ and $\left(x-\Delta_{x}\right)-\left(z-\Delta_{z}\right)$ planes of the translated coordinate system. In this sense the angles $\Theta_{x}$ and $\Theta_{y}$ represent skew angles of transverse angular misalignments.

Using Eq. (14), the coordinate transformation in Eq. (9) can be written in component form as

$$
\begin{aligned}
& x^{\prime}=x-\Delta_{x}-\Theta_{x}\left(z-\Delta_{z}\right), \\
& y^{\prime}=y-\Delta_{y}-\Theta_{y}\left(z-\Delta_{z}\right), \\
& z^{\prime}=z-\Delta_{z}+\Theta_{x} x+\Theta_{y} y .
\end{aligned}
$$

Similarly, the field transform (12) in component form is

$$
\begin{aligned}
& B_{x}=B_{x}^{\prime}+\Theta_{x} B_{z}^{\prime}, \\
& B_{y}=B_{y}^{\prime}+\Theta_{y} B_{z}^{\prime}, \\
& B_{z}=B_{z}^{\prime}-\Theta_{x} B_{x}^{\prime}-\Theta_{y} B_{y}^{\prime} .
\end{aligned}
$$

Inserting the ideal solenoid field description in Eq. (2) (change all field components and coordinates to primed) in Eq. (16), and then applying the coordinate transform (15) in the result, the field in the laboratory frame can be expressed as

$$
\begin{aligned}
& B_{x}=-\frac{1}{2} \frac{\partial B_{z 0}^{\prime}(s)}{\partial s}\left[x-\Delta_{x}-\Theta_{x}\left(z-\Delta_{z}\right)\right]+\Theta_{x} B_{z 0}^{\prime}(s) \\
& B_{y}=-\frac{1}{2} \frac{\partial B_{z 0}^{\prime}(s)}{\partial s}\left[y-\Delta_{y}-\Theta_{y}\left(z-\Delta_{z}\right)\right]+\Theta_{y} B_{z 0}^{\prime}(s) \\
& B_{z}=B_{z}^{\prime}(s)+\frac{1}{2} \frac{\partial B_{z 0}^{\prime}(s)}{\partial s} \Theta_{x}\left[x-\Delta_{x}-\Theta_{x}\left(z-\Delta_{z}\right)\right]+\frac{1}{2} \frac{\partial B_{z 0}^{\prime}(s)}{\partial s} \Theta_{y}\left[y-\Delta_{y}-\Theta_{y}\left(z-\Delta_{z}\right)\right] .
\end{aligned}
$$

Here, $s=z^{\prime}=z-\Delta_{z}+\Theta_{x} x+\Theta_{y} y$ is the axial coordinate.

The laboratory frame expression (17) for the fields of the translated and rotated solenoid will be applied to derive linear equations of motion in Sec. 3. In this context, both the transverse coordinate excursions in $x$ and $y$ and the misalignment parameters $\Delta_{x}, \Delta_{y}, \Delta_{z}, \Theta_{x}$, and $\Theta_{y}$ are expected to be small. Therefore, Eq. (17) can be Taylor expanded in all small quantities and secondorder (i.e, terms proportional to $\xi \eta$, with $\xi$ and $\eta$ each representing one of the expanded transverse coordinates or the misalignment parameters) and higherorder terms can be dropped. Carrying out this procedure, we calculate the leading order magnetic field for a misaligned solenoid as

$$
\begin{aligned}
& B_{x}(\mathbf{x})=-\frac{1}{2} \frac{\partial B_{z 0}(z)}{\partial z}\left(x-\Delta_{x}-\Theta_{x} z\right)+B_{z 0}(z) \Theta_{x} \\
& B_{y}(\mathbf{x})=-\frac{1}{2} \frac{\partial B_{z 0}(z)}{\partial z}\left(y-\Delta_{y}-\Theta_{y} z\right)+B_{z 0}(z) \Theta_{y} \\
& B_{z}(\mathbf{x})=B_{z 0}(z)-\frac{\partial B_{z 0}(z)}{\partial z} \Delta_{z} .
\end{aligned}
$$

Here, we have eliminated the primes in the function $B_{z 0}(z)$, which we henceforth take to be the function defining the ideal, aligned solenoid. Equation (18) represents the leading-order field of an ideal solenoid described by Eq. (2) which 
is transversely misaligned with displacement parameters $\Delta_{x}, \Delta_{y}, \Delta_{z}$, and rotational parameters $\Theta_{x}$ and $\Theta_{y}$. Equation (18) shows that the misalignments generate $z$-varying transverse dipole fields in addition to an effective shift in the longitudinal field component.

In addition to beam focusing solenoids, the lattice may also contain steering dipoles to enable bending of the centroid in the $x$ - and $y$-planes to steer the centroid back on-axis. To model a steering dipole that generates a field primarily along the $x$-axis, we simply take

$$
B_{x}(\mathbf{x})=B_{x}(z)
$$

with $B_{x}(z)$ a prescribed function set by the geometry and excitation of the dipole. An analogous description is taken for dipoles that generate a field oriented along the $y$-axis. Axial field components associated with the $z$-variation are neglected. Because the dipoles are generally of limited strength and are intended to weakly bend the centroid trajectory to correct for small misalignment induced centroid excursions, effects of mechanical misalignments of the dipole on the centroid can typically be neglected as higher-order when deriving centroid equations of motion.

\section{Centroid model}

We consider a beam composed of charged particles of mass $m$ and charge $q$ and abbreviate the phase-space coordinates of the transverse beam centroid as

$$
\begin{array}{rlrl}
x & \equiv\langle x\rangle_{\perp}, & x^{\prime} & \equiv\left\langle x^{\prime}\right\rangle_{\perp}, \\
y \equiv\langle y\rangle_{\perp}, & y^{\prime} & \equiv\left\langle y^{\prime}\right\rangle_{\perp} .
\end{array}
$$

Here, primes denote derivatives with respect to the axial coordinate $s$ of the centroid in the lattice (not to be confused with the coordinate transforms used only in Sec. 2) and $\langle\cdots\rangle_{\perp}$ denotes a statistical average over the transverse phase-space of the beam. The transverse centroid slice propagates with rigidity $[B \rho]=m \gamma_{b} \beta_{b} c / q$, where $c$ is the speed of light in vacuo, and $\beta_{b}=$ const and $\gamma_{b}=$ $\sqrt{1-\beta_{b}^{2}}$ are the axial relativistic factors of the coasting centroid. Neglecting radiation effects, and image charge and current forces, the transverse equations of motion of the centroid $\mathbf{x}_{\perp}(s)=x(s) \hat{\mathbf{x}}+y(s) \hat{\mathbf{y}}$ evolving in a magnetic field $\mathbf{B}(\mathbf{x})$ can be expressed within the paraxial approximation as $[11,9]$

$$
\begin{aligned}
x^{\prime \prime} & =-\frac{B_{y}}{[B \rho]}+\frac{B_{z}}{[B \rho]} y^{\prime}, \\
y^{\prime \prime} & =\frac{B_{x}}{[B \rho]}-\frac{B_{z}}{[B \rho]} x^{\prime} .
\end{aligned}
$$

The focusing lattice is formed from a linear superposition of (possibly misaligned) focusing solenoids and ideal steering dipoles. Consistent with Eq. (2), 
the solenoids have ideal (meaning with no mechanical misalignments and perfect axisymmetry) axial field

$$
B_{z}(s)=\sum_{j=1}^{N_{s}} B_{s j} F_{s j}\left(s-s_{j}\right),
$$

and ideal transverse field

$$
\mathbf{B}_{\perp}(s)=B_{x}(s) \hat{\mathbf{x}}+B_{y}(s) \hat{\mathbf{y}}=-\frac{1}{2} \sum_{j=1}^{N_{s}} B_{s j} F_{s j}^{\prime}\left(s-s_{j}\right) \mathbf{x}_{\perp} .
$$

Here, $j=1,2, \cdots, N_{s}$ is the solenoid index, $B_{s j}$ is the peak axial field of $j$ th solenoid, $F_{s j}(z)$ is the axial fringe function of the $j$ th solenoid with normalization chosen such that $F_{s j}(0)=1, s=s_{j}$ is the axial center of the $j$ th solenoid in the focusing lattice, and $F_{s j}^{\prime}\left(s-s_{j}\right)=\partial F_{s j}(z) /\left.\partial z\right|_{z=s-s_{j}}$. The lattice can include several types of solenoids because the functions $F_{s j}(z)$ can be different for every solenoid $(j)$. If the lattice is periodic, then all $F_{s j}$ are identical, and the centers $s_{j}$ are uniformly spaced. For an ideal, linear solenoid, the function $F_{s j}$ depends only on geometric parameters of the solenoid and the strength $B_{s j}$ is set by the current excitation in the solenoid coil.

Using a similar notation to that employed for the solenoids, $x$-plane dipole fields for beam steering are linearly superimposed and are denoted by

$$
B_{x}(s)=\sum_{j=1}^{N_{x}} B_{x j} F_{x j}\left(s-s_{j}\right) .
$$

Here, $N_{x}$ is the number of $x$-field dipoles, $B_{x j}$ is the peak field of the $j$ th dipole, $F_{x j}(s)$ with $F_{x j}(0)=1$ is the fringe function of the $j$ th dipole, and $s_{j}$ is the axial center of the $j$ th dipole. Note that $s_{j}$ can refer to the center of the $j$ th solenoid or dipole, with the distinction being clear from the context of the fringe function $F_{s j}$ or $F_{x j}$ referencing the center. An expression analogous to Eq. (24) applies to $y$-field dipoles for beam steering. The $x$-field dipoles bend the centroid in the $y$-plane, and the $y$-field dipoles bend the centroid in the $x$-plane.

To describe the applied fields of a lattice with misaligned solenoids, we modify Eqs. (22) and (23) consistent with the expression in Eq. (18). These misaligned solenoid fields and the $x$ - and $y$-plane dipole fields [see Eq. (24)] are linearly superimposed and inserted in the centroid equations of motion (21). Terms generated that are proportional to $\Delta_{z} x^{\prime}$ and $\Delta_{z} y^{\prime}$ are neglected as higher order. The resulting linear particle equations of motion can be expressed in complex form as

$$
\begin{aligned}
\underline{z}^{\prime \prime}+i\left(\sum_{j=1}^{N_{s}} \mathcal{S}_{j} F_{s j}\right) \underline{z}^{\prime}+i\left(\sum_{j=1}^{N_{s}} \frac{\mathcal{S}_{j}}{2} F_{s j}^{\prime}\right) \underline{z}= & i \sum_{j=1}^{N_{s}}\left[\frac{\mathcal{S}_{j}}{2} F_{s j}^{\prime} \underline{\Delta}_{j}+\frac{\mathcal{S}_{j}}{2} F_{s j}^{\prime} \underline{\Theta}_{j}\left(s-s_{j}\right)+\mathcal{S}_{j} F_{s j} \underline{\Theta}_{j}\right] \\
& +i \sum_{j=1}^{N_{x}} \mathcal{D}_{x j} F_{x j}-\sum_{j=1}^{N_{y}} \mathcal{D}_{y j} F_{y j} .
\end{aligned}
$$


Here, $i=\sqrt{-1}, \underline{z} \equiv x+i y$ is the complex coordinate of the centroid,

$$
\begin{aligned}
& \underline{\Delta}_{j} \equiv \Delta_{x j}+i \Delta_{y j}, \\
& \underline{\Theta}_{j} \equiv \Theta_{x j}+i \Theta_{y j},
\end{aligned}
$$

are complex displacement and rotational misalignment parameters of the $j$ th solenoid of the lattice, and

$$
\mathcal{S}_{j} \equiv \frac{B_{s j}}{[B \rho]}, \quad \mathcal{D}_{x j} \equiv \frac{B_{x j}}{[B \rho]}, \quad \mathcal{D}_{y j} \equiv \frac{B_{y j}}{[B \rho]},
$$

are scaled solenoid $\left(\mathcal{S}_{j}\right)$ and dipole $\left(\mathcal{D}_{x j}\right.$ and $\left.\mathcal{D}_{y j}\right)$ strength parameters. In Eq. (25) and henceforth we make notational abbreviations such as $F_{s j} \equiv F_{s j}(s-$ $\left.s_{j}\right), F_{s j}^{\prime} \equiv \partial F_{s j}(z) /\left.\partial z\right|_{z=s-s_{j}}$. Underbars are also employed to distinguish complex-valued quantities from real-valued quantities.

If the misalignment parameters $\underline{\Delta}_{j}$ and $\underline{\Theta}_{j}$ are known or negligible (i.e., $\underline{\Delta}_{j} \simeq 0$ and $\underline{\Theta}_{j} \simeq 0$ ), then the equation of motion (25) can be integrated in $s$ from specified initial $\left(s=s_{i}\right)$ conditions $\underline{z}\left(s_{i}\right)=x\left(s_{i}\right)+i y\left(s_{i}\right)$ and $\underline{z}^{\prime}\left(s_{i}\right)=$ $x^{\prime}\left(s_{i}\right)+i y^{\prime}\left(s_{i}\right)$ to calculate the evolution of the centroid orbit in $\underline{z}-\underline{z}^{\prime}$ phasespace. Note that there is no dependence of the leading-order orbit described by Eq. (25) on the longitudinal displacement misalignments $\Delta_{z j}$ of the solenoid magnets.

The equation of motion (25) can be expressed in simplified form by transforming to "Larmor" frame undergoing a local $s$-varying transverse rotation[7, $8,9]$. We denote coordinates in the Larmor frame with tildes and take

$$
\underline{z}=\underline{\tilde{z}} e^{i \tilde{\psi}(s)},
$$

where $\tilde{\psi}(s)$ is a real-valued phase-function that specifies the angle of rotation. Using this rotational transform and taking

$$
\tilde{\psi}^{\prime}=-\frac{1}{2} \sum_{j=1}^{N_{s}} \mathcal{S}_{j} F_{s j}
$$

leads to a simplified expression of the equations of motion (25) in the Larmor frame,

$$
\begin{aligned}
\underline{\tilde{z}}^{\prime \prime}+\tilde{\kappa} \underline{\tilde{z}} & =i \sum_{j=1}^{N_{s}}\left[\frac{\mathcal{S}_{j}}{2} F_{s j}^{\prime} \underline{\Delta}_{j}+\frac{\mathcal{S}_{j}}{2} F_{s j}^{\prime} \underline{\Theta}_{j}\left(s-s_{j}\right)+\mathcal{S}_{j} F_{s j} \underline{\Theta}_{j}\right] e^{-i \tilde{\psi}} \\
& +i \sum_{j=1}^{N_{x}} \mathcal{D}_{x j} F_{x j} e^{-i \tilde{\psi}}-\sum_{j=1}^{N_{y}} \mathcal{D}_{y j} F_{y j} e^{-i \tilde{\psi}}
\end{aligned}
$$

Here,

$$
\tilde{\kappa}(s) \equiv \frac{1}{4}\left[\sum_{j=1}^{N_{s}} \mathcal{S}_{j} F_{s j}\left(s-s_{j}\right)\right]^{2}
$$


is the lattice focusing function in the Larmor frame. Equation (31) can be equivalently expressed as $\kappa=\left(k_{L}\right)^{2}$, where $k_{L}=q B_{z}\left(\mathbf{x}_{\perp}=0\right) /\left(2 \gamma_{b} \beta_{b} m c\right)$ is the Larmor-frame focusing wavenumber. Without loss of generality, we take a reference angle $\tilde{\psi}\left(s_{i}\right)=0$, and the solution to Eq. (29) for $\tilde{\psi}$ is

$$
\tilde{\psi}(s)=-\frac{1}{2} \sum_{j=1}^{N_{s}} \mathcal{S}_{j} \int_{s_{i}}^{s} d \bar{s} F_{s j}\left(\bar{s}-s_{j}\right) .
$$

Phase-space coordinates of the centroid in the laboratory and Larmor frames are related by

$$
\begin{aligned}
\underline{z} & =\underline{\tilde{z}} e^{i \tilde{\psi}}, \\
\underline{z}^{\prime} & =\left(\underline{\tilde{z}}^{\prime}+i \tilde{\psi}^{\prime} \underline{\tilde{z}}\right) e^{i \tilde{\psi}},
\end{aligned}
$$

with the inverse transform

$$
\begin{aligned}
\underline{\tilde{z}} & =\underline{z} e^{-i \tilde{\psi}} \\
\underline{\tilde{z}}^{\prime} & =\left(\underline{z}^{\prime}-i \tilde{\psi}^{\prime} \underline{z}\right) e^{-i \tilde{\psi}} .
\end{aligned}
$$

These transforms are applied with $\tilde{\psi}^{\prime}$ and $\tilde{\psi}$ specified by Eqs. (29) and (32). To solve for the centroid evolution in the Larmor frame, $\tilde{\psi}$ is specified in Eq. (32) and Eq. (30) is integrated from the initial $\left(s=s_{i}\right)$ conditions

$$
\begin{aligned}
\underline{\tilde{z}}\left(s_{i}\right) & =\underline{z}\left(s_{i}\right), \\
\underline{\tilde{z}}^{\prime}\left(s_{i}\right) & =\underline{z}^{\prime}\left(s_{i}\right)-i \tilde{\psi}^{\prime}\left(s_{i}\right) \underline{\tilde{z}}\left(s_{i}\right) .
\end{aligned}
$$

The initial angle $\underline{\tilde{z}}^{\prime}\left(s_{i}\right)$ in Eq. (35) has been compensated due to the local rotation of the Larmor frame even though $\tilde{\psi}\left(s=s_{i}\right) \equiv 0$.

Direct integration of the linear, complex-form centroid equations of motion for misaligned solenoids will not, in general, be possible either in the laboratory frame [see Eq. (25)] or in the Larmor frame [see Eq. (30)] because the displacement and rotational misalignment parameters $\underline{\Delta}_{j}$ and $\underline{\Theta}_{j}$ of the solenoids are not a priori known. However, the dependence of the equations on the misalignment parameters is linear. This linearity can be exploited to define auxiliary complex "alignment" functions that characterize changes in centroid properties under general small-amplitude misalignments. This development is analogous to the usual construction of the "dispersion" function used to characterize changes in single-particle orbit properties within a periodic lattice due to small deviations in particle momentum[10]. The alignment functions are more complicated than the dispersion function because each focusing optic can be individually misaligned in both a translational and rotational sense and the centroid is also Larmor rotating in the solenoidal focusing system. Analogously to the case of the alignment functions, "bending" functions will also be introduced to exploit the linearity of the equations in the the steering dipole strength parameters $\mathcal{D}_{x j}$ and $\mathcal{D}_{y j}$. 
To solve the Larmor-frame centroid equation of motion (30) for general misalignments we expand the linear orbit as follows. First we resolve the solution $\underline{\tilde{z}}$ as

$$
\begin{aligned}
\underline{\tilde{z}}(s) & =\underline{\tilde{z}}_{h}(s)+\underline{\tilde{z}}_{p}(s), \\
\underline{\tilde{z}}^{\prime}(s) & =\underline{\tilde{z}}_{h}^{\prime}(s)+\underline{\tilde{z}}_{p}^{\prime}(s),
\end{aligned}
$$

where $\underline{\tilde{z}}_{h}(s)$ is the general solution to the homogeneous (zero misalignments) equation of motion

$$
\underline{\tilde{z}}_{h}^{\prime \prime}+\tilde{\kappa} \underline{\tilde{z}}_{h}=0
$$

and $\underline{\tilde{z}}_{p}(s)$ is any particular solution to Eq. (30) with $\underline{\tilde{z}}=\underline{\tilde{z}}_{p}$. Equation (37) describing $\underline{z}_{h}$ is the usual Hill's equation[10,9] describing single-particle orbits in complex form and Eq. (30) for $\underline{\tilde{z}}_{p}$ corresponds to Hill's equation on the LHS with misalignment and bending driving terms on the RHS. In this formulation we are free to simplify the choice of initial conditions in the solution of the particular solution for $\underline{\tilde{z}}_{p}$ because the homogeneous solution $\underline{\tilde{z}}_{h}$ can assume the necessary modified initial conditions to obtain the correct solution for $\underline{\tilde{z}}=\underline{\tilde{z}}_{h}+\underline{\tilde{z}}_{p}$. Without loss in generality, the linearity of the particular solution can be further exploited to expand $\underline{\tilde{z}}_{p}(s)$ as

$$
\begin{aligned}
& \underline{\tilde{z}}_{p}=\sum_{j=1}^{N_{s}}\left(\underline{\Delta}_{j} \underline{\tilde{D}}_{j}+\underline{\Theta}_{j} \underline{\tilde{R}}_{j}\right)+\sum_{j=1}^{N_{x}} \mathcal{D}_{x j} \underline{\tilde{B}}_{x j}+\sum_{j=1}^{N_{y}} \mathcal{D}_{y j} \underline{\tilde{B}}_{y j}, \\
& \underline{\tilde{z}}_{p}^{\prime}=\sum_{j=1}^{N_{s}}\left(\underline{\Delta}_{j} \underline{\tilde{D}}_{j}^{\prime}+\underline{\Theta}_{j} \underline{\tilde{R}}_{j}^{\prime}\right)+\sum_{j=1}^{N_{x}} \mathcal{D}_{x j} \underline{\tilde{B}}_{x j}^{\prime}+\sum_{j=1}^{N_{y}} \mathcal{D}_{y j} \underline{\tilde{B}}_{y j}^{\prime},
\end{aligned}
$$

Here, $\underline{\tilde{D}}_{j}$ is a "displacement alignment function" satisfying

$$
\underline{\tilde{D}}_{j}^{\prime \prime}+\tilde{\kappa} \underline{\tilde{D}}_{j}=\frac{i}{2} \mathcal{S}_{j} F_{s j}^{\prime} e^{-i \tilde{\psi}},
$$

subject to the initial $\left(s=s_{i}\right)$ conditions $\underline{\tilde{D}}_{j}\left(s_{i}\right)=0=\underline{\tilde{D}}_{j}^{\prime}\left(s_{i}\right), \underline{\tilde{R}}_{j}$ is a "rotational alignment function" satisfying

$$
\underline{\tilde{R}}_{j}^{\prime \prime}+\tilde{\kappa} \underline{\tilde{R}}_{j}=\frac{i}{2} \mathcal{S}_{j} F_{s j}^{\prime}\left(s-s_{j}\right) e^{-i \tilde{\psi}}+i \mathcal{S}_{j} F_{s j} e^{-i \tilde{\psi}},
$$

subject to the initial $\left(s=s_{i}\right)$ conditions $\underline{\tilde{R}}_{j}\left(s_{i}\right)=0=\underline{\tilde{R}}_{j}^{\prime}\left(s_{i}\right)$, and $\underline{\tilde{B}}_{x j}$ and $\underline{\tilde{B}}_{y j}$ are the $x$ - and $y$-field steering dipole "bending functions" satisfying

$$
\begin{aligned}
& \underline{\tilde{B}}_{x j}^{\prime \prime}+\tilde{\kappa} \underline{\tilde{B}}_{x j}=i F_{x j} e^{-i \tilde{\psi},}, \\
& \underline{\tilde{B}}_{y j}^{\prime \prime}+\tilde{\kappa} \underline{\tilde{B}}_{y j}=-F_{y j} e^{-i \tilde{\psi}},
\end{aligned}
$$

subject to the initial $\left(s=s_{i}\right)$ conditions $\underline{\tilde{B}}_{x j}\left(s_{i}\right)=0=\underline{\tilde{B}}_{x j}^{\prime}\left(s_{i}\right)$ and $\underline{\tilde{B}}_{y j}\left(s_{i}\right)=$ $0=\underline{\tilde{B}}_{y j}^{\prime}\left(s_{i}\right)$. Generally, outside of simple limiting cases, Eqs. (39)-(41) will 
need to be integrated numerically. There are $j=1,2, \cdots, N_{s}$ complexvalued displacement and rotational misalignment functions $\underline{\tilde{D}}_{j}(s)$ and $\underline{\tilde{R}}_{j}(s)$ - one each for every solenoid. Each complex-valued function represent two real-valued functions through it's associated real and imaginary components. Unfortunately, this complexity is necessary because each solenoid has two displacement $\left(\underline{\Delta}_{j}=\Delta_{x}+i \Delta_{y}\right)$ and two rotational $\left(\underline{\Theta}_{j}=\Theta_{x}+i \Theta_{y}\right)$ misalignment parameters which can, in the small-amplitude approximations made, influence the centroid in an independent way. Similarly, there are $j=1,2, \cdots, N_{x}$ and $j=1,2, \cdots, N_{y}$ complex-valued bending functions $\underline{\tilde{B}}_{x j}$ and $\underline{\tilde{B}}_{y j}$ that represent the action of the $x$ - and $y$-field component steering dipoles. The LHS of Eqs. (39)-(41) are expressions of Hill's equation in the Larmor frame and the complex exponential factors $e^{-i \tilde{\psi}}$ in the RHS terms project bending dipole fields arising from solenoid misalignments [Eqs. (39) and (40)] and steering dipoles [Eq. (41)] into the Larmor frame. The linear structure of the equations has been exploited to separate amplitudes of solenoid misalignment errors $\left(\underline{\Delta}_{j}\right.$ and $\left.\underline{\Theta}_{j}\right)$ and the steering dipole strengths $\left(\mathcal{D}_{x j}\right.$ and $\left.\mathcal{D}_{y j}\right)$ from the corresponding alignment and bending functions as defined. This separation is analogous to the one employed in usual definitions of momentum spread related "dispersion" functions $[10,9]$ and allows isolation of factors contributing to the evolution of the beam centroid.

Because zero value initial conditions imposed on the solenoid alignment functions and the dipole bending functions (i.e., all $\underline{\tilde{D}}_{j}, \underline{\tilde{D}}_{j}^{\prime}, \underline{\tilde{R}}_{j}, \underline{\tilde{R}}_{j}^{\prime}, \underline{\tilde{B}}_{x j}, \underline{\tilde{B}}_{x j}^{\prime}, \underline{\tilde{B}}_{y j}$, $\underline{\tilde{B}}_{y j}^{\prime}$ zero at $s=s_{i}$ ), the initial conditions of the homogeneous solution $\underline{\tilde{z}}_{h}$ to Eq. (37) must absorb any initial centroid misalignment, i.e.,

$$
\begin{aligned}
& \underline{\tilde{z}}_{h}\left(s_{i}\right)=\underline{\tilde{z}}\left(s_{i}\right), \\
& \underline{\tilde{z}}_{h}^{\prime}\left(s_{i}\right)=\underline{\tilde{z}}^{\prime}\left(s_{i}\right) .
\end{aligned}
$$

The homogeneous phase-space solution to Eq. (37) consistent with these initial conditions can be expressed in terms of a transfer map as[10, 9]

$$
\left[\begin{array}{l}
\underline{\tilde{z}}_{h}(s) \\
\underline{\tilde{z}}_{h}^{\prime}(s)
\end{array}\right]=\left[\begin{array}{ll}
\tilde{C}\left(s \mid s_{i}\right) & \tilde{S}\left(s \mid s_{i}\right) \\
\tilde{C}^{\prime}\left(s \mid s_{i}\right) & \tilde{S}^{\prime}\left(s \mid s_{i}\right)
\end{array}\right] \cdot\left[\begin{array}{c}
\underline{\tilde{z}}_{h}\left(s_{i}\right) \\
\underline{\tilde{z}}_{h}^{\prime}\left(s_{i}\right)
\end{array}\right] .
$$

Here, $\tilde{C}\left(s \mid s_{i}\right)$ and $\tilde{S}\left(s \mid s_{i}\right)$ are real-valued cosine- and sine-like principal orbit functions that satisfy the Hill's equation

$$
\tilde{F}^{\prime \prime}+\tilde{\kappa} \tilde{F}=0,
$$

with $\tilde{F}=\tilde{C}\left(s \mid s_{i}\right)$ or $\tilde{S}\left(s \mid s_{i}\right)$ satisfying the initial conditions $\tilde{C}\left(s_{i} \mid s_{i}\right)=1=$ $\tilde{S}^{\prime}\left(s_{i} \mid s_{i}\right)$ and $\tilde{C}^{\prime}\left(s_{i} \mid s_{i}\right)=0=\tilde{S}\left(s_{i} \mid s_{i}\right)$. The $\tilde{C}$ and $\tilde{S}$ are real-valued functions because the real and imaginary components of $\underline{\tilde{z}}_{h}$ are decoupled in Eq. (44). As with the alignment functions, both $\tilde{C}$ and $\tilde{S}$ depend only on the ideal lattice structure and are independent of any misalignments. The solution in Eq. (43) represents the solenoid oscillation in the ideal system from the initial conditions (42) in the absence of any solenoid misalignments (i.e., $\tilde{\Delta}_{j}=0=\underline{\Theta}_{j}$ ) or 
dipole steering corrections (i.e., $\mathcal{D}_{x j}=0=\mathcal{D}_{y j}$ ). In general, the cosine- and sine-like solutions $\tilde{C}$ and $\tilde{S}$ must be numerically calculated. However, for lattices with piecewise constant $\tilde{\kappa}$, the solutions can be analytically calculated in terms of standard special functions[10, 11, 9]. Because the decoupled real and imaginary components of $\tilde{z}_{h}=\tilde{x}_{h}+i \tilde{y}_{h}$ satisfy the same Hill's equation (43) (i.e., $\tilde{x}_{h}^{\prime \prime}+\tilde{\kappa} \tilde{x}_{h}=0$ and $\tilde{y}_{h}^{\prime \prime}+\tilde{\kappa} \tilde{y}_{h}=0$ ), the usual Courant-Snyder and Wronskian invariants of the equation[10, 13,9] can be individually applied to both $\tilde{x}_{h}$ and $\tilde{y}_{h}$ to better understand properties of the homogeneous solution $\underline{\tilde{z}}_{h}$.

The homogeneous and particular solution expansion can be applied to express phase-space coordinates of the centroid at axial location $s$ as

$$
\begin{aligned}
\underline{\tilde{z}}(s)=\underline{\tilde{z}}\left(s_{i}\right) \tilde{C}\left(s \mid s_{i}\right)+\underline{\tilde{z}}^{\prime}\left(s_{i}\right) \tilde{S}\left(s \mid s_{i}\right) & +\sum_{j=1}^{N_{s}}\left[\underline{\Delta}_{j} \underline{\tilde{D}}_{j}(s)+\underline{\Theta}_{j} \underline{\tilde{R}}_{j}(s)\right] \\
& +\sum_{j=1}^{N_{x}} \mathcal{D}_{x j} \underline{\tilde{B}}_{x j}(s)+\sum_{j=1}^{N_{y}} \mathcal{D}_{y j} \underline{\tilde{B}}_{y j}(s), \\
\underline{\tilde{z}}^{\prime}(s)=\underline{\tilde{z}}\left(s_{i}\right) \tilde{C}^{\prime}\left(s \mid s_{i}\right)+\underline{\tilde{z}}^{\prime}\left(s_{i}\right) \tilde{S}^{\prime}\left(s \mid s_{i}\right) & +\sum_{j=1}^{N_{s}}\left[\underline{\Delta}_{j} \underline{\tilde{D}}_{j}^{\prime}(s)+\underline{\Theta}_{j} \underline{\tilde{R}}_{j}^{\prime}(s)\right] \\
& +\sum_{j=1}^{N_{x}} \mathcal{D}_{x j} \underline{\tilde{B}}_{x j}^{\prime}(s)+\sum_{j=1}^{N_{y}} \mathcal{D}_{y j} \underline{\tilde{B}}_{y j}^{\prime}(s) .
\end{aligned}
$$

This Larmor-frame expansion of the centroid solution can be projected back to the laboratory frame using the transformation in Eq. (33) to obtain

$$
\begin{aligned}
\underline{z}(s) & =\underline{z}\left(s_{i}\right)\left[\tilde{C}\left(s \mid s_{i}\right)-i \tilde{\psi}\left(s_{i}\right) \tilde{S}\left(s \mid s_{i}\right)\right] e^{i \tilde{\psi}(s)}+\underline{z}^{\prime}\left(s_{i}\right) \tilde{S}\left(s \mid s_{i}\right) e^{i \tilde{\psi}(s)} \\
& +\sum_{j=1}^{N_{s}}\left[\underline{\Delta}_{j} \underline{\tilde{D}}_{j}(s)+\underline{\Theta}_{j} \underline{\tilde{R}}_{j}(s)\right] e^{i \tilde{\psi}(s)}+\sum_{j=1}^{N_{x}} \mathcal{D}_{x j} \underline{\tilde{B}}_{x j}(s) e^{i \tilde{\psi}(s)}+\sum_{j=1}^{N_{y}} \mathcal{D}_{y j} \tilde{\underline{B}}_{y j}(s) e^{i \tilde{\psi}(s)}, \\
\underline{z}^{\prime}(s)= & \underline{z}\left(s_{i}\right)\left[\tilde{C}^{\prime}\left(s \mid s_{i}\right)-i \tilde{\psi}^{\prime}\left(s_{i}\right) \tilde{S}^{\prime}\left(s \mid s_{i}\right)+i \tilde{\psi}^{\prime}(s) \tilde{C}\left(s \mid s_{i}\right)+\tilde{\psi}^{\prime}\left(s_{i}\right) \tilde{\psi}^{\prime}(s) \tilde{S}\left(s \mid s_{i}\right)\right] e^{i \tilde{\psi}(s)} \\
& +\underline{z}^{\prime}\left(s_{i}\right)\left[\tilde{S}^{\prime}\left(s \mid s_{i}\right)+i \tilde{\psi}^{\prime}(s) \tilde{S}\left(s \mid s_{i}\right)\right] e^{i \tilde{\psi}(s)} \\
& +\sum_{j=1}^{N_{s}}\left[\underline{\Delta}_{j} \tilde{\tilde{D}}_{j}^{\prime}(s)+\underline{\Theta}_{j} \underline{\tilde{R}}_{j}^{\prime}(s)\right] e^{i \tilde{\psi}(s)}+i \tilde{\psi}^{\prime}(s) \sum_{j=1}^{N_{s}}\left[\underline{\Delta}_{j} \underline{\tilde{D}}_{j}(s)+\underline{\Theta}_{j} \underline{\tilde{R}}_{j}(s)\right] e^{i \tilde{\psi}(s)} \\
& +\sum_{j=1}^{N_{x}} \mathcal{D}_{x j} \underline{\tilde{B}}_{x j}^{\prime}(s) e^{i \tilde{\psi}(s)}+i \tilde{\psi}^{\prime}(s) \sum_{j=1}^{N_{x}} \mathcal{D}_{x j} \underline{\tilde{B}}_{x j}(s) e^{i \tilde{\psi}(s)} \\
& +\sum_{j=1}^{N_{y}} \mathcal{D}_{y j} \underline{\tilde{B}}_{y j}^{\prime}(s) e^{i \tilde{\psi}(s)}+i \tilde{\psi}^{\prime}(s) \sum_{j=1}^{N_{y}} \mathcal{D}_{y j} \underline{\tilde{B}}_{y j}(s) e^{i \tilde{\psi}(s)} .
\end{aligned}
$$

Here we have also employed Eq. (35) to express the initial conditions $\underline{\tilde{z}}\left(s_{i}\right)$ and $\underline{\tilde{z}}^{\prime}\left(s_{i}\right)$ in the laboratory frame.

\section{Applications of centroid expansion}

The utility of the centroid expansion derived in Sec. 3 is illustrated by formulating algorithms to: statistically analyze centroid oscillations in response to an 
ensemble of errors (Sec. 4.1), calculate actual solenoid misalignment parameters based on experimental measurements of the centroid (Sec. 4.2), and optimally manipulate the beam centroid using dipole steering fields (Sec. 4.3). In these applications, a machine operating point refers to a distinct set of solenoid strengths $\left\{\mathcal{S}_{j}\right\}$ [see Eq. (27)].

\subsection{Statistical Analysis of Expected Centroid Oscillations}

The centroid expansion (46) can be applied with zero steering terms (i.e., $\left.\mathcal{D}_{x j}=0=\mathcal{D}_{y j}\right)$ to efficiently calculate initial centroid errors tolerances [i.e., the range of $\underline{z}\left(s_{i}\right)=x\left(s_{i}\right)+i y\left(s_{i}\right)$ and $\left.\underline{z}^{\prime}\left(s_{i}\right)=x^{\prime}\left(s_{i}\right)+i y^{\prime}\left(s_{i}\right)\right]$ and mechanical alignment tolerances of the solenoids (i.e., the range of $\Delta_{j}=\Delta_{x j}+i \Delta_{y j}$ and $\left.\underline{\Theta}_{j}=\Theta_{x j}+i \Theta_{y j}\right)$ necessary to keep centroid excursions within specified bounds. The principal orbit functions $(\tilde{C}$ and $\tilde{S})$ and the alignment functions $\left(\underline{\tilde{D}}_{j}\right.$ and $\underline{\tilde{R}}_{j}$ ) need only be calculated once for the operating point of the ideal machine lattice. Because the Larmor transformation back to the laboratory frame do not the magnitude of the centroid excursions [i.e., $|\underline{z}|=|\tilde{z}|$, see Eq. (33)], maximum excursions can be calculated directly from the Larmor-frame expression in Eq. (45).

Distributions of initial centroid and alignment parameters can be taken such as uniform or, more realistically, truncated Gaussian distributions, and then the corresponding distribution of centroid excursions is obtained directly from Eq. (46). If more realistic, truncated error distributions are employed (e.g., distributions with finite upper bound errors such as truncated Gaussian or uniform distributions), then maximum centroid excursions can be calculated as a function of $s$ to bound conceivable centroid evolutions. Maximum excursions coupled with rms measures of expected statistical ensemble excursions can provide a good understanding of likely, uncorrected centroid excursions and the buildup of oscillation amplitudes as more misaligned elements are traversed. If the error distributions are statistically independent with zero mean value, then contributions to the rms deviations calculated over the statistical ensemble will add in quadrature (i.e., statistical average $x^{2}$ can be calculated from statistical average $x^{2}$ due to displacements only, plus statistical average $x^{2}$ due to rotations only, ...). This can be exploited to help understand the relative importance of error classes (e.g., displacement or rotational solenoid misalignments) contributing to expected centroid excursions.

\subsection{Calculation of Lattice Misalignment Parameters}

The centroid coordinates $x, x^{\prime}, y$, and $y^{\prime}$ are typically only measurable at a limited number of diagnostic locations with fixed axial coordinates in the lattice. For a given machine operating point, one full centroid phase-space measurement at a single diagnostic location provides four independent numbers. This data is insufficient to uniquely constrain the misalignment parameters of $N_{s}$ solenoids "upstream" of the measurement because the $j=1,2, \cdots, N_{s}$ solenoids have $4 N_{s}$ misalignment parameters $\underline{\Delta}_{j}=\Delta_{x j}+i \Delta_{y j}$ and $\underline{\Theta}_{j}=\Theta_{x j}+i \Theta_{y j}$ and there are (possibly) four parameters associated with any initial centroid offset [i.e., 
nonzero components of $\underline{z}\left(s_{i}\right)=x\left(s_{i}\right)+i y\left(s_{i}\right)$ and $\left.\underline{z}^{\prime}\left(s_{i}\right)=x^{\prime}\left(s_{i}\right)+i y^{\prime}\left(s_{i}\right)\right]$, if unknown. However, sufficient independent data at a single diagnostic location can be generated by varying the machine operating point. Changes in operating point are effected by varying the scaled solenoid strengths $\mathcal{S}_{j}=B_{s j} /[B \rho]$ by either adjusting field strengths $B_{s j}$ of the solenoids or by adjusting the particle rigidity $[B \rho]$ via variations in the kinetic energy or changing the particle species.

With sufficient measurement data measured for constraint, the expansion in Sec. 3 can be applied to calculate the misalignment data. We illustrate this with a simple illustrative example that can be straightforwardly modified to apply to other situations. Assume that dipole steering is not employed $\left(\mathcal{D}_{x j}=0=\mathcal{D}_{y j}\right)$ and there is a single diagnostic location at $s=s_{m}$ where all four centroid coordinates $x, y, x^{\prime}$ and $y^{\prime}$ can be measured. The measured centroid data is transformed into the Larmor frame using Eq. (34). Centroid phase-space measurements are made at $s=s_{m}$ for $N$ sets of independent solenoid strengths $\mathcal{S}_{j}$ that are sufficiently different to result in significant centroid variations between operating point measurements relative to measurement uncertainties. We employ a superscript $k=1,2, \cdots, N$ to denote quantities associated with the $N$ sets of solenoid strengths and abbreviate expressions following patterns such as $\underline{\tilde{z}}_{m}^{k} \equiv \underline{\tilde{z}}^{k}\left(s_{m}\right)$. The Larmor frame centroid expansion (45) and Eq. (35) (to express the initial conditions in the laboratory frame) can be applied to arrange the centroid measurement data $\left(\underline{\tilde{z}}_{m}^{k}\right.$ and $\left.\underline{\tilde{z}}_{m}^{\prime k}\right)$, alignment function values at the measurement point $\left(\underline{\tilde{D}}_{j m}^{k}\right.$ and $\left.\underline{\tilde{R}}_{j m}^{k}\right)$, unknown initial centroid $\left[\underline{z}_{i}\left(s_{i}\right)=\underline{z}_{i}\right.$ and $\underline{z}^{\prime}\left(s_{i}\right)=\underline{z}_{i}^{\prime}$ with $\left.s_{i}<s_{m}\right]$, and misalignment parameters $\left(\underline{\Delta}_{j}\right.$ and $\left.\underline{\Theta}_{j}\right)$ in matrix form as

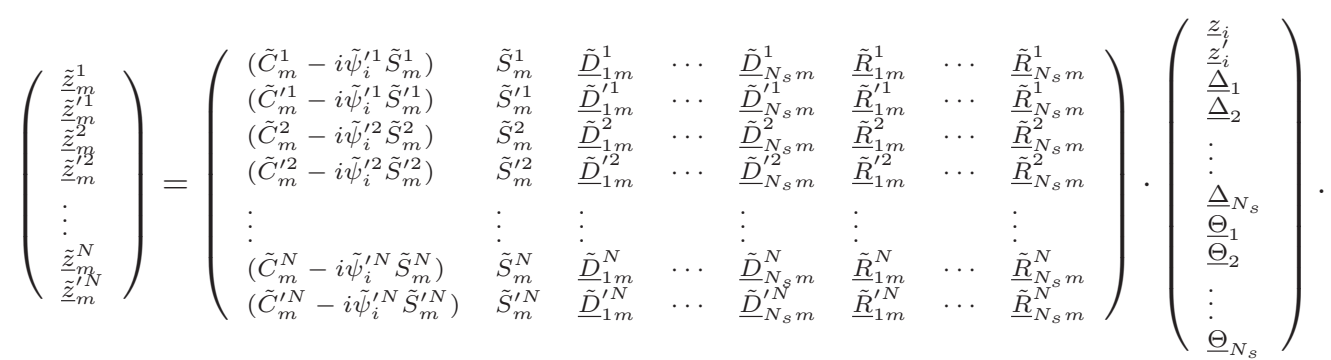

We express this equation in abbreviated form as

$$
\underline{\tilde{\mathbf{Z}}}=\underline{\tilde{\mathbf{A}}} \cdot \underline{\mathbf{D}} \text {. }
$$

Here, $\underline{\tilde{\mathbf{Z}}}$ is a complex-valued vector of length $2 N$ composed of measured centroid data for the operating point sets, $\underline{\tilde{\mathbf{A}}}$ is complex-valued matrix of dimension $(2 N) \times\left(2 N_{s}+2\right)$ composed of principal orbit and alignment functions evaluated at the measurement plane for the excitation sets, and $\underline{\mathbf{D}}$ is a complex-valued vector of length $2 N_{s}+2$ composed of the unknown initial centroid complex coordinates in the laboratory frame and the unknown solenoid misalignment parameters. The notation $\tilde{\psi}_{i}^{k} \equiv \tilde{\psi}^{k}\left(s_{i}\right)$ denotes the initial Larmor phase associated with the $k$ th set of solenoid strengths at $s=s_{i}$. It should be stressed 
that Eq. (47) should only be applied in situations where the initial centroid conditions $\underline{z}_{i}$ and $\underline{z}_{i}^{\prime}$ do not vary with the $N$ sets of solenoid strengths. This generally requires that the initial condition at $s=s_{i}$ be upstream of the axial fringe fields of the solenoids analyzed and that none of the solenoid strengths upstream of $s=s_{i}$ are varied in changing the operating points.

If $2 N=2 N_{s}+2$ and the $N$ data points represent independent sets of solenoid strengths $\mathcal{S}_{j}$, then the matrix system is square and can be uniquely solved for the vector of initial centroid offsets and solenoid misalignment parameters $\underline{\mathbf{D}}$ as

$$
\underline{\mathbf{D}}=\underline{\tilde{\mathbf{A}}}^{-1} \cdot \underline{\tilde{\mathbf{Z}}} \text {. }
$$

Here, $\underline{\tilde{\mathbf{A}}}^{-1}$ is the inverse matrix of $\underline{\tilde{\mathbf{A}}}$. If $2 N>2 N_{s}+2$, then the measurement data will overconstrain $\underline{\mathbf{D}}$. If measurements are reliable, the overconstrained data is consistent to small measurement uncertainties. In such cases, a singular value decomposition (SVD) can be applied to generate a pseudo-inverse of $\underline{\mathbf{A}}$ for the overconstrained system[14]. The solution for $\underline{\mathbf{D}}$ in this case is given by Eq. (49) with $\underline{\tilde{\mathbf{A}}}^{-1}$ calculated employing SVD constructs. The solution so defined represents the correct solution for the measurement data in a minimum least-square error sense[14].

If the initial centroid is aligned at $s=s_{i}$, then the components of $\underline{\mathbf{D}}$ associated with the initial centroid offset and the components of $\underline{\tilde{\mathbf{A}}}$ associated with the principal orbit functions (i.e., $\tilde{C}_{m}^{1}, \tilde{S}_{m}^{1}$, etc.) can be removed to simplify the formulation. Such a case applies if $s_{i}$ corresponds to a location where the centroid was last corrected to measurement accuracy, or where the centroid is by definition aligned (for example, if the centroid emerging from an injector is used to define the machine centerline).

In a long lattice with many focusing elements, large numbers of centroid measurements will be necessary to sufficiently constrain $\underline{\mathbf{D}}$. Reducing the size of the problem generally increases the accuracy that the misalignment parameters are found. This follows because lesser number of parameters result in a more simple correlation of variations in measured centroid coordinates with variations in the machine operating point. The problem size can be reduced by turning off $\left(\mathcal{S}_{j} \neq 0\right)$ the maximum number of solenoids possible in the lattice to remove them from the inversion. After the misalignments of the excited solenoids are determined, the solenoids first turned off can be energized and (to the extent possible) solenoids previously energized can be turned off and the process repeated to determine the remaining misalignment parameters. Such procedures can be limited though due to the need to maintain transverse beam confinement within the machine aperture. Use of "pencil" type beams with small transverse extent produced via aperturing or a special injector can aid in reducing the number of solenoids that must be energized to maintain confinement.

\subsection{Centroid Steering}

If the misalignment data determined using the procedures outlined in Sec. 4.2, the lattice can be corrected to null the centroid phase-space coordinates $x, x^{\prime}$, 
$y$, and $y^{\prime}$ by physically moving the solenoids (realigning) to correct the mechanical alignment errors. Alternatively, or in addition to this, if sufficient numbers of steering dipoles are placed in the lattice, their strengths $\mathcal{D}_{x j}$ and $\mathcal{D}_{y j}$ can be set to correct (null) the centroid phase-space errors errors at specified axial positions in the lattice or to purposefully steer the centroid off-axis consistent with he needs of specific applications. Generally, at least two axially separated dipoles acting in each independent transverse plane (four total correction dipoles) will be necessary to place in the lattice upstream of the axial location where centroid steering is needed. More dipoles allows flexibility in tuning (see later discussions). Generally, steering dipoles should be placed before, but axially close to the position where the corrections are needed to avoid accumulation of further errors. Iron-free designs of steering dipoles can have radially nested coils to produce superimposed $x$ - and $y$-field components in one element and thereby reduce the number of steering modules necessary to insert in the lattice. Because centroid oscillations tend to grow in amplitude in a random walk sense (i.e., with the square root of the number of focusing solenoids traversed), correcting the centroid at regular intervals helps to control the amplitude of centroid excursions. Effective control of amplitude growth also suppresses nonlinear effects (images and applied fields) that scale with centroid amplitude to improve the validity of the linear model.

The centroid expansion (46) can be applied to efficiently calculate dipole strengths $\mathcal{D}_{x j}$ and $\mathcal{D}_{y j}$ needed to steer the beam centroid. As with the case of the calculation of misalignment parameters outlined in Sec. 4.2, details of steering procedures vary strongly with the specific lattice and application in question and it is not practical to cover all possibilities. Therefore, we outline several examples to illustrate typical correction procedures. It is straightforward to adapt these procedures to address specific lattices and applications of interest.

Consider a specific machine operating point and the case where the phasespace of the centroid is measured at a single diagnostic location at $s=s_{m}$. Denote the measured, complex-form phase-space coordinates in the absence of steering fields $\left(\mathcal{D}_{x j}=0=\mathcal{D}_{y j}\right)$ by $\underline{z}_{m}=x\left(s_{m}\right)+i y\left(s_{m}\right)$ and $\underline{z}_{m}^{\prime}=x^{\prime}\left(s_{m}\right)+$ $i y^{\prime}\left(s_{m}\right)$ and corresponding "target" complex-form phase-space coordinates at $s=s_{m}$ by $\underline{z}_{t}$ and $\underline{z}_{t}^{\prime}$. The measured and target centroid coordinates transformed to the Larmor frame are denoted $\underline{\tilde{z}}_{m}, \underline{\tilde{z}}_{m}^{\prime}$, and $\underline{\tilde{z}}_{t}, \underline{\tilde{z}}_{t}^{\prime}$. Assume that there are two $(j=1,2)$ steering dipoles with axial location $s_{j}<s_{m}$ placed in each plane with fields oriented along the $x$ - and $y$-axes and that the centroid is to be steered from the measured to the target values at $s=s_{m}$ by setting the dipole strengths $\mathcal{D}_{x j}$ and $\mathcal{D}_{y j}$ accordingly. Equation (45) is applied to this situation to obtain

$$
\begin{aligned}
& \underline{\tilde{z}}_{m}=\underline{\tilde{z}}\left(s_{i}\right) \tilde{C}\left(s_{m} \mid s_{i}\right)+\underline{\tilde{z}}^{\prime}\left(s_{i}\right) \tilde{S}\left(s_{m} \mid s_{i}\right)+\sum_{j=1}^{N_{s}}\left[\underline{\Delta}_{j} \underline{\tilde{D}}_{j}\left(s_{m}\right)+\underline{\Theta}_{j} \underline{\tilde{R}}_{j}\left(s_{m}\right)\right], \\
& \underline{\tilde{z}}_{m}^{\prime}=\underline{\tilde{z}}\left(s_{i}\right) \tilde{C}^{\prime}\left(s_{m} \mid s_{i}\right)+\underline{\tilde{z}}^{\prime}\left(s_{i}\right) \tilde{S}^{\prime}\left(s_{m} \mid s_{i}\right)+\sum_{j=1}^{N_{s}}\left[\underline{\Delta}_{j} \underline{\tilde{D}}_{j}^{\prime}\left(s_{m}\right)+\underline{\Theta}_{j} \underline{\tilde{R}}_{j}^{\prime}\left(s_{m}\right)\right],
\end{aligned}
$$

and, assuming that the misalignment and initial condition do not change with 
with the Dipole steering fields (i.e., $\underline{\tilde{z}}_{m}$ and $\underline{\tilde{z}}_{m}^{\prime}$ constants),

$$
\begin{aligned}
& \underline{\tilde{z}}_{t}=\underline{\tilde{z}}_{m}+\sum_{j=1}^{2} \mathcal{D}_{x j} \underline{\tilde{B}}_{x j}\left(s_{m}\right)+\sum_{j=1}^{2} \mathcal{D}_{y j} \underline{\tilde{B}}_{y j}\left(s_{m}\right) \\
& \underline{\tilde{z}}_{t}^{\prime}=\underline{\tilde{z}}_{m}^{\prime}+\sum_{j=1}^{2} \mathcal{D}_{x j} \underline{\tilde{B}}_{x j}^{\prime}\left(s_{m}\right)+\sum_{j=1}^{2} \mathcal{D}_{y j} \underline{\tilde{B}}_{y j}^{\prime}\left(s_{m}\right)
\end{aligned}
$$

Using this result, the equations of constraint are then separated into real $(\operatorname{Re}[\cdots])$ and imaginary $(\operatorname{Im}[\cdots])$ parts and expressed in matrix form as

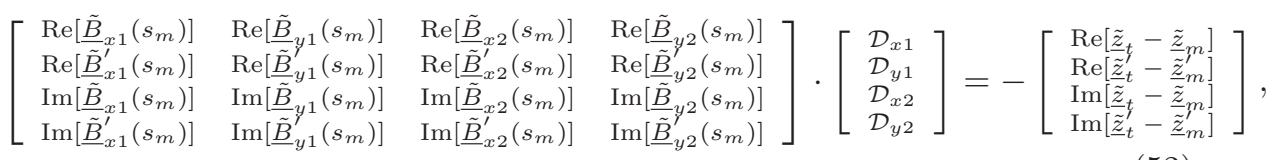

which we abbreviate as

$$
\tilde{\mathbf{B}} \cdot \mathcal{D}=-\tilde{\Delta \mathbf{Z}} .
$$

Here, $\tilde{\mathbf{B}}$ is the real matrix of dimension $4 \times 4$ composed of the real and imaginary parts of the complex bending functions evaluated at $s=s_{m}, \mathcal{D}$ is a real vector of length 4 composed of (unknown) steering dipole strengths necessary to zero the centroid, and $\tilde{\Delta \mathbf{Z}}$ is a real vector of length 4 composed of the real and imaginary parts of the difference between target and measured complex centroid coordinates in the Larmor frame evaluated at $s=s_{m}$. Equation (53) can be uniquely solved for the unknown steering strengths $\mathcal{D}$ as

$$
\mathcal{D}=-\tilde{\mathbf{B}}^{-1} \cdot \tilde{\Delta \mathbf{Z}}
$$

where $\tilde{\mathbf{B}}^{-1}$ is the inverse matrix of $\tilde{\mathbf{B}}$.

Steering dipole strengths $\mathcal{D}_{x j}$ and $\mathcal{D}_{y j}$ set according to Eq. (54) with $\underline{\tilde{z}}_{t}=$ $0=\underline{\tilde{z}}_{t}^{\prime}$ will zero the centroid at $s=s_{m}$. Because $\tilde{\boldsymbol{\Delta} \mathbf{Z}}$ reflects centroid evolution due to alignment errors of any solenoids placed between or after the dipole steering elements, the correction does not neglect the accumulation of additional errors that the centroid experiences after the deflections associated with the first steering elements in the correction pairs are applied. In this sense the correction can be regarded as optimal. If more than two dipoles per plane are employed to correct the centroid, then a similar analysis as presented above applies, but the steering dipole amplitudes will not be fully constrained for a unique solution. This residual freedom in the steering amplitudes can be exploited to reduce the magnitude of required steering fields $\left|B_{x j}\right|$ and $\left|B_{y j}\right|$ and/or limit centroid excursions while undergoing steering corrections.

If the misalignment parameters are known, then the formulation above can be applied with $\underline{\tilde{z}}_{m}$ and $\underline{\tilde{z}}_{t}^{\prime}$ given by Eq. (50) rather than experimental measurements. In this case the equation can also be applied at arbitrary values of axial coordinate $s$ downstream of the steering dipoles (i.e., $s_{m} \rightarrow s$ with $s_{m}>s_{j}$ ). This result can also be applied to efficiently calculate the range of steering strengths $\left(\mathcal{D}_{x j}\right.$ and $\left.\mathcal{D}_{y j}\right)$ necessary to correct expected distribution of mechanical alignment $\left(\underline{\Delta}_{j}\right.$ and $\left.\underline{\Theta}_{j}\right)$ and initial centroid $\left[\underline{\tilde{z}}\left(s_{i}\right)\right.$ and $\left.\underline{\tilde{z}}^{\prime}\left(s_{i}\right)\right]$ errors. 


\section{CONCLUSIONS}

A linear, small-amplitude description of centroid orbit was derived to expand the centroid orbit in terms misalignment and steering driven terms superimposed on the ideal centroid evolution in the perfectly aligned system. Properties of this expansion were exploited to formulate efficient procedures for optimal centroid steering corrections and calculation of system (beam and solenoid) misalignment parameters based on usual experimental centroid measurements.

Procedures formulated will typically require shot-to-shot reproducibility of the system. Also, misalignment properties of the system from any mechanical motion of lattice components must evolve slowly over the time that the correction procedure is carried out. Accuracy of the corrections can be expected to decrease if centroid orbit excursions reach a large fraction of the magnet apertures due to nonlinear applied field and image charge forces not included in the model becoming stronger. Corrections can be applied iteratively to reduce such effects and improve accuracy.

Analogous procedures to those developed here can be more simply formulated and applied to quadrupole transport lattices due to the lack of $x-y$ coupling in the ideal lattice. The model presented can also be applied to systems with acceleration if results are interpreted in terms of appropriate canonical variables[9]. Errors in solenoid and dipole strength, or equivalently the centroid rigidity, result in more substantial changes in analysis. Models presented are presently being applied to analyze the solenoidal transport lattice in the Neutralized Drift Compression Experiment at the Lawrence Berkeley National Laboratory[1]. Results obtained will be reported in future work.

\section{ACKNOWLEDGMENTS}

The authors wish to thank J.J. Barnard, I. Haber, and L. Prost for useful discussions. C.J. Wootton (U.C. Berkeley undergraduate, Nuclear Engineering Department) was tragically killed before this paper was complete. This research was performed under the auspices of the U.S. Department of Energy at the Lawrence Livermore and Lawrence Berkeley National Laboratories under Contracts No. DE-AC52-07NA27344 and No. DE-AC02-05CH11231.

\section{References}

[1] P.A. Seidl et. al, submitted for publication, Nuclear Inst. Meth. A, 2008.

[2] A. C. Paul, G. J. Caporaso, Y.-J. Chen, Y. J. Chen, G. Westenskow, W. M. Fawley, E. P. Lee, The beamline for the second axis of the dual axis radiographic hydrodynamic test facility, in: Proceedings of the 1999 Particle Accelerator Conference, New York City, NY, IEEE Piscataway, NJ 08855, 1999, pp. THP126, 3254. 
[3] A. Burov, S. Nagaitsev, A. Shemyakin, Y. Derbenev, Optical principles of beam transport for relativistic electron cooling, Phys. Rev. Special Topics - Accel. and Beams 3 (2000) 094002.

[4] S. Nagaitsev, D. Broemmelsiek, A. Burov, K. Carlson, C. Gattuso, M. Hu, T. Kroc, L. Prost, S. Pruss, M. Sutherland, C. W. Schmidt, A. Shemyakin, V. Tupikov, A. Warner, G. Kazakevich, S. Seletskiy, Experimental Demonstration of Relativistic Electron Cooling, Phys. Rev. Lett. 96 (2006) 044801.

[5] Y.-J. Chen, Corkscrew Modes in Linear Accelerators, Nuc. Instr. Meth. A 292 (1990) 455.

[6] Y.-J. Chen, Control of Transverse Motion Caused by Chromatic Abberation and Misalignments in Linear Accelerators, Nuc. Instr. Meth. A 398 (1997) 139 .

[7] H. Wiedemann, Particle Accelerator Physics II: Nonlinear and HigherOrder Beam Dynamics, Springer-Verlag, New York, 1995.

[8] S. M. Lund, B. Bukh, Stability Properties of the Transverse Envelope Equations Describing Intense Ion Beam Transport, Phys. Rev. Special Topics Accelerators and Beams 7 (2004) 024801.

[9] See U. S. Particle Accelerator School lectures notes archived on http://uspas.fnal.gov/ for: J. J. Barnard and S. M. Lund, "Beam Physics with Intense Space Charge," Annapolis Maryland School sponsored by the University of Maryland at College Park, June 16-27, 2008.

[10] H. Wiedemann, Particle Accelerator Physics: Basic Principles and Linear Beam Dynamics, Springer-Verlag, New York, 1993.

[11] M. Reiser, Theory and Design of Charged Particle Beams, John Wiley \& Sons, Inc., New York, 1994.

[12] E.P. Lee and M. Leitner, "Solenoidal Fields for Ion Beam Transport and Focusing," LBNL-6317, Lawrence Berkeley National Laboratory (2007).

[13] G. Franchetti, Linear Beam Optics in Solenoidal Channels, Phys. Rev. Special Topics - Accelerators and Beams 4 (2001) 074001.

[14] W. H. Press, B. P. Flannery, S. A. Teukolsky, W. A. Vetterling, Numerical Recipes in FORTRAN 77: The Art of Scientific Computing, second edition Edition, Cambridge University Press, New York, 1992. 